\title{
CONTRA EL FUEGO EN EL BURGOS DEL SETECIENTOS
}

\author{
Against fire in the Eighteenth-Century Burgos
}

\author{
Francisco José Sanz de la Higuera ${ }^{1}$ \\ sanzdelahiguera@gmail.com \\ profesor EEMM jubilado. España \\ Fecha de recepción: 26/11/2020 \\ Fecha de aceptación: 03/03/2021
}

RESUMEN: Los incendios ocurridos en el caserío de la ciudad de Burgos en el devenir del siglo XVIII hicieron imprescindible la adquisición y mantenimiento de una bomba contra el fuego por parte del Ayuntamiento. La construcción de las casas con madera, la quema de carbón y leña en las chimeneas y braseros en los interiores domésticos y la existencia de industrias potencialmente peligrosas en la ciudad pre-industrial hacían preciso, a todas luces, disponer de un sistema anti-incendios eficaz, rápido y funcional con el que atajar el grave peligro que se cernía sobre la población.

Palabras clave: Bomba de incendios; Incendios; Ayuntamiento; Burgos; Siglo XVIII.

ABSTRACT: The fires that occurred in the hamlet of the city of Burgos in the course of the 18th century made it essential for the City Council to acquire and maintain a fire pump. The construction of the houses with wood, the burning of coal and firewood in the fireplaces and braziers indoors and the existence of potentially dangerous industries in the pre-industrial city made it necessary, clearly, to have an effective, fast and functional anti-fire system to tackle the serious danger that was looming over the population.

Keywords: Fire pump; Fires; Town Hall; Burgos, 18th century.

Sumario: 1. Introducción, fuentes documentales y aproximación bibliográfica. 2. De la «porcion de Cantaros y algunas achas» a la "Bomba para cortar los Yncendios» en el Burgos del Setecientos. 3. El origen del "Bonbero» y sus cometidos en el siglo XVIII burgalés. 4. A modo de conclusión. 5 . Referencias bibliográficas.

\footnotetext{
1 https://orcid.org/0000-0002-0210-4001.
} 


\section{INTRODUCCIÓN, FUENTES DOCUMENTALES Y BIBLIOGRAFÍA}

En los Regimientos ordinarios del 21 de abril y 28 de abril de 1768, uno de los temas "para tratar y resolver» por los dirigentes del Concejo de Burgos fue la propuesta de «la Compra de una Bomba para cortar los Yncendios que ocurran en esta Ciudad», artefacto al que se añadían "todos los peltrechos anexos a la expda Bomba y las Palas y Picos que se contemplan prezisos para cortar y extinguir dhos Yncendios ${ }^{2}$. Se inicia, a partir de ese momento, un proceso de adquisición de una máquina y las «herramientas» complementarias, que culminó el 25 de febrero de 1773. Se hizo presente el «haberse entregado ya la Bomba conducida desde la villa de Bilbao, con parte de los Peltrechos necesarios para su manejo en los Casos de incendios qe ocurran ${ }^{3}$.

En estas páginas se efectúa una reconstrucción de varias problemáticas. Descuellan, en primera instancia, el cómo el Concejo dotó a la Ciudad de dicho artefacto; en segundo término, el dónde estaba ubicado; en tercer lugar, el quién se encargó de su mantenimiento y, a la postre, el cuánto se desembolsó, a lo largo de la centuria, tanto para la adquisición y el cuidado de dicha bomba como para la extinción de las "Quemas» que se produjeron en el tejido urbano burgalés en el devenir del Setecientos. La fuente documental esencial para llevar a cabo tales quehaceres históricos se encuentra custodiada en el Archivo Municipal de Burgos en su versión original. Es preciso distinguir entre los libros de Acuerdos (Actas) de Gobierno (1700-1804), los libros Acuerdos (Actas) de Abastos (1771-1804) y los libros de Acuerdos (Actas) de la Junta de Propios y Arbitrios (1768-1804)4. Además, contamos con varios documentos sueltos. En primera instancia, Las «Providencias del Sor Yntendente Respectibas a la Quema que acaeció en la Calle de La Puebla: la noche del día doce a el trece del presente mes de Abril» $»^{5}$. En segundo término, la venta, a Prudencio Valderrama, del «sitio donde se colocaba la bomba» ${ }^{6}$ y un documento «Sobre componer la bomba del Ayuntamto pa apagar incendios» (1849)7.

2 Archivo Municipal de Burgos (AMB). Actas de Gobierno (AG). Regimiento ordinario (RO) del 21 De abril de 1768, folio140r y del 28 de abril de 1768, folios 149r-150v.

${ }^{3}$ AMB. AG. RO del 25 de febrero de 1773, folio 50v.

${ }^{4}$ Los gruesos volúmenes de papel, encuadernados con borrego, son accesibles, aunque no es recomendable, en la sala de investigadores, si bien lo acertado es manejar la versión microfilmada, en la tecnología apropiada, habilitada en dicha sala. Empero, todas las Actas se encuentran digitalizadas, en formato PDF, y resulta muy sencillo, y gratificante, su descarga. Es de agradecer al Ayuntamiento de Burgos, y en concreto a las directoras y empleadas del Archivo Municipal de Burgos su amable y siempre eficaz profesionalidad y diligencia en la atención a los investigadores.

${ }^{5}$ AMB. Policía Urbana (PU). Bernardo Alonso de Illera. Histórica. Legajo 22-686 (17 de abril de 1768), sin foliar.

${ }^{6}$ AMB. Julián Álvarez. Histórica. Legajo 11-38 (7 de febrero de 1799), sin foliar.

${ }^{7}$ AMB. José Angulo. Histórica. Legajo 22-360 (13 de abril de 1849), sin foliar. 
Esta documentación constituye una poderosa herramienta para profundizar en la historia urbana en la España del siglo XVIII, en problemáticas que han tenido, hasta ahora, un escaso desarrollo historiográfico y bibliográfico ${ }^{8}$.

La bibliografía sobre dichas temáticas, las bombas para la extinción de incendios y estos en sí mismos, no es muy abundante. Descuellan, en especial, aunque citados sin un ánimo exhaustivo ni la realización de un estado de la cuestión, los análisis vertidos en las obras de Sanz de la Higuera (2013) ${ }^{9}$, Cebreiros Álvarez (1997), Vidal Rivas (2012), Yuste González (2015), Sanz Yagüe (2012), Rupérez Almajano (1987), Anero Cárcamo (2007), Campos y Saez (1984), Garnot (1989) o López Giménez (2012).

\section{DE LA "PORCION DE CANTAROS Y ALGUNAS ACHAS" A LA "BOMBA PARA CORTAR LOS YNCENDIOS" EN EL BURGOS DEL SETECIENTOS}

Estas páginas suponen un recorrido crítico por un proceso histórico preñado de ironías. La reconstrucción histórica suele conjugar la búsqueda de respuestas a los interrogantes de las hipótesis con la, a veces impredecible, aparición de imponderables historiográfico, derivados del contenido de la documentación aprehendida.

¿Por qué en 1768 ? El corregidor e Intendente Bañuelos ${ }^{10}$, ante el cariz del incendio acaecido en la madrugada del 12 al 13 de abril de dicho año, elevó al Concejo la imperiosa necesidad de adquirir una «Bomba para cortar los Yncendios que ocurran en esta ciudad». Las intervenciones de los regidores perpetuos discurrieron por la asunción de la propuesta, enfatizando lo «Util e indispensable [de] la Compra de la enunciada Bomba, sus Peltrechos y erramientas para cortar los Yncendios" y que el erario municipal, los Propios, se hiciera cargo del desembolso, «sin necesidad de hacerse repartimto alguno sobre los Dueños de dhas Casas, por los embarazos que ocurrirán para su exacción y lo odioso que sería, siempre que resulte haver fondos suficientes para dha Compra» ${ }^{11}$. Todos los presentes en aquel Regimiento eran conscientes, por una parte, de que la única forma de llegar a ejecutar la compra

8 Sin un ánimo exhaustivo, descuellan, entre otras, las propuestas desarrolladas por Belmonte López Huici, Cuesta Martínez, García Cano y Pozas Poveda, 1987; García Ruipérez, 1999 o Muñoz Bort, 1986.

${ }^{9}$ En este artículo aparece un acervo bibliográfico de notable utilidad para el seguimiento de los incendios en los siglos XVII y XVIII.

10 Sobre Bañuelos y sus quehaceres profesionales en la Corregiduría y la Intendencia, véanse, entre otros, los análisis de Abbad, F. y Ozanam, 1992; Abbad, 1982; Sánchez Domingo, 1996 y Coll Coll, 2015. Bañuelos fue Intendente de Galicia (La Coruña) entre 1782, con 66 años, y 1792, fecha en la que se jubiló, a los 76 años. Falleció en octubre de 1802, con 84 años.

${ }^{11}$ AMB. AG. RO del 28 de abril de 1768, folio 149r. Sobre las dificultades económicas de los Concejos en el Setecientos véanse, por ejemplo, Torres Sánchez, 1999; Gutiérrez Alonso, 1997 y Jarque Martínez, 2017. 
pasaba, «con la posible brevedad», por, den primera instancia, como era preceptivo, solicitar «la facultad correspte de los Señores del Real y Supremo Consejo de Castilla» ${ }^{12} y$, por otra, en último término, que, una vez adquirida la bomba, era imprescindible lograr su «veneficio y conservación ${ }^{13}$.

La deriva de los incendios ocurridos en la ciudad -Gráfico I- era, sin duda, un acicate esencial en el estímulo de este desembolso. La construcción de las casas con madera, la quema de carbón y leña en las chimeneas y braseros en los interiores domésticos ${ }^{14}$ y la existencia de industrias potencialmente peligrosas en la ciudad pre-industrial generaban un diagnóstico preñado de temores y prevenciones. Los vecinos de la ciudad se enfrentaban a múltiples problemáticas. Una de ellas devenía

de que a muchos años se alla Con notable falta de Agua de la que Antigua mente tenía dha fuente y sus nazim ${ }^{\text {tos }}$, de donde Benía mayor abundanzia, [que] Se allan Perdidos, ocasionado de los Pocos Medios que an Padezido y Padezen dhos Vezinos y juntam ${ }^{\text {te }}$ Con el desconsuelo de tan poco agua, que si llegase a subzeder algún caso fortuito de inzendio, lo que Dios no quiera, por la distanzia [a] Que se allan los Ríos de dha Vezindad padezerían muchas casas ${ }^{15}$.

A las carencias de agua se añadía el que, para quienes trabajaban «personalmente para cortar el fuego" que se desataba, de vez en cuando, en las casas particulares o en edificios públicos, la Ciudad únicamente tenía prevenidos "porción de Cántaros y algunas achas ${ }^{16}$. Con tan escasos, y deficitarios, medios se enfrentaron los incendios sufridos en 1705 en la "Casa de los miradores» de la Plaza Mayor ${ }^{17}$, en 1721 en la cárcel Real, "azia la parte q cae al calabozo» ${ }^{18}$, en 1730 en una casa del Mercado Mayor, propiedad de la Ciudad ${ }^{19}$ y en 1768 en la calle La Puebla ${ }^{20}$.

12 Véanse los análisis y propuestas de de Castro, 2019 y Andújar Castillo y Ponce, Leiva, 2018.

${ }^{13}$ AMB. AG. RO del 28 de abril de 1768, folio 149v.

${ }^{14}$ Sanz de la Higuera, 2020. En aquellos tiempos, las casas, y las calles, se iluminaban con antorchas o con velas, además de un surtido conjunto de candeleros, palmatorias, bujías, candiles, etcétera. Véanse Negueruela Superviola, 2011 y Sanz de la Higuera, 2013. El fuego también se utilizaba, por supuesto, para calentar las estancias, con braseros y/o chimeneas, y para cocinar. Era habitual observar en las ciudades pequeños incendios como consecuencia de puntuales accidentes, que, en ocasiones, se complicaban.

${ }^{15}$ AMB. AG. RO del 2 de diciembre de 1712, folio $264 \mathrm{v}$.

${ }^{16}$ AMB. AG. RO del 9 de agosto de 1742, folio 216r.

17 AMB. AG. RO del 23 de noviembre de 1705, folio 250r.

${ }^{18}$ AMB. AG. RO del 17 de febrero de 1721, folio 61r.

19 AMB. AG. RO del 2 de diciembre de 1730, folio 298v.

${ }^{20}$ AMB. Policía Urbana (PU). Bernardo Alonso de Illera. Histórica. Legajo 22-686 (17 de abril de 1768), sin foliar. 


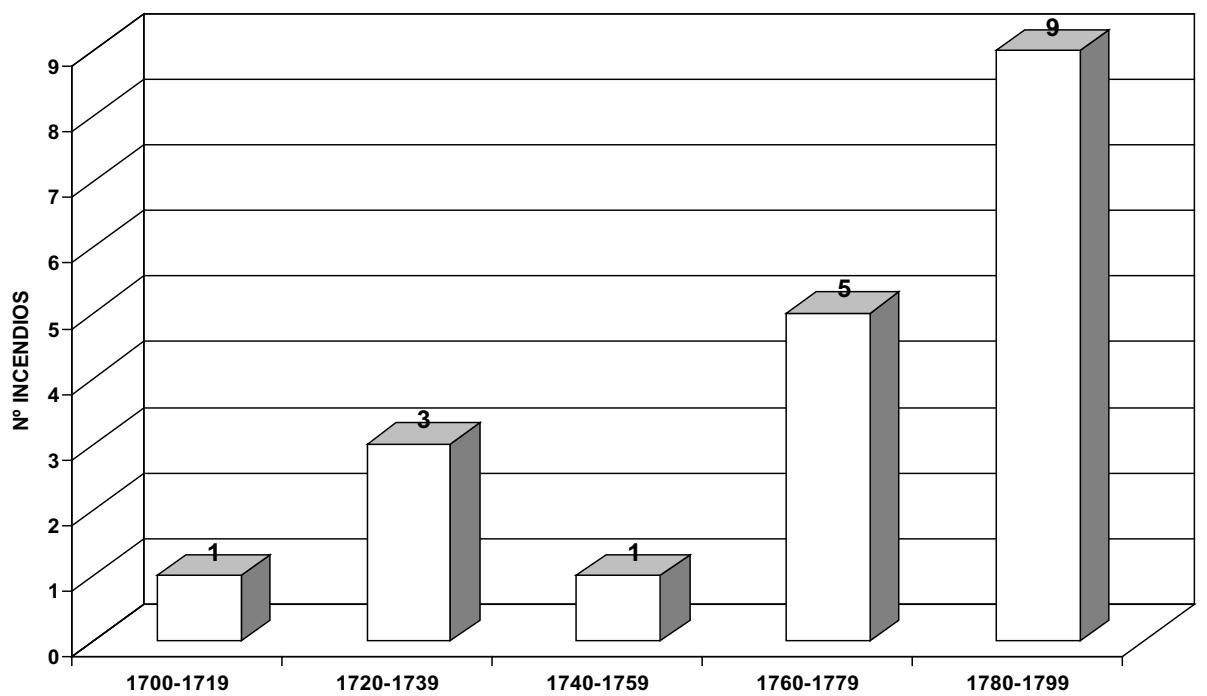

Fuente documental: AMB. AG, AA y JPA. Múltiples años (1700-1799).

En Burgos no se pecó de vanguardistas ni de innovadores ex novo. Una surtida muestra de ejemplos demuestra que anteriormente otros territorios y otras localidades ya habían introducido, en su combate contra el fuego de los incendios, el usufructo de bombas manuales para combatir las llamas. Sin entrar en precedentes excesivamente remotos ${ }^{21}$, enfatizar que, en 1518, el alemán Anthony Blatner construye, en Augsburgo, el primer carro de bomberos -durante la Edad Media la extinción de los incendios había sido siempre un auténtico caos y cada vecino tenía que organizarse por su cuenta o esperar a que el señor feudal de turno tomara las riendas- (Historia de los bomberos, 2020).

En España, en 1497, en Valladolid una treintera de moriscos, los llamados «moros llamados a matar el fuego", armados de diferentes herramientas, se dedicaban a sofocar los fuegos urbanos. «En 1515, Juana I de Castilla emitió una Cédula Real por la que se estableció lo que podríamos denominar el primer cuerpo de bomberos oficial» (Bravo, 2019). En tiempos de Felipe II lo habitual era disponer de «un

${ }^{21}$ Probablemente, fuera Ctesibius de Alejandría es inventor de la bomba contra incendios, a mediados del siglo II a. C. Véase "La evolución de los carros de bomberos en EE. UU.», (2020). Es creíble que el primer servicio de bomberos organizado se dio en la antigua Roma. En el año 22 a. C., Cesar Augusto organizó el primer equipo de bomberos de la historia, formado por esclavos que se llamaban "vigiles», si bien, "En realidad, el primer servicio de bomberos lo organizó Craso cuando aún formaba parte del triunvirato con Cesar y Pompeyo, unos años antes». Véase "Historia de los bomberos» (2020). 
azadón, un hacha y una palanca de hierro». En Valladolid, en concreto, se habían dotado de "herradas de cuero, jeringas (bombas de mano), escaleras y algunos otros "aparejos" ». Una Real Cédula de Felipe II de 1561 establecía que hubiese personas que velasen de noche el cuidado del material, lo que supone ya un bien organizado servicio de incendios. En Oviedo, el Concejo tomaba, en 1571, el acuerdo de enviar a Bilbao un encargado de adquirir «jeringas gruesas y buenas, para que con ellas haya preparo para algún inzendio o peligro de fuego». Sin duda, la villa vizcaína era especialista en esos artefactos -como vamos a comprobar en las próximas páginas(Lampérez y Romea, 2012).

En el siglo XVI se detecta una prodigiosa proposición de inventos ${ }^{22}$ que persiste, también, en el Seiscientos ${ }^{23}$. Hitos esenciales en este proceso fueron 1655, 1668 y 1773 . En 1655, en Nuremberg, se construye la primera máquina (una bomba de mano) para la lucha contra incendios (Anero Cárcamo, 2007, p. 7). El Ayuntamiento de Ámsterdam encargó a Jan van der Heyden la invención de una bomba contra incendios. En 1672, inventó, en efecto, la primera manguera flexible de cuero, con una longitud de hasta 15 metros, y conexiones de latón. Heyden había logrado notoriedad con el diseño, en 1633, de una "manguera» aplicada a la extinción de incendios, que fue mejorando progresivamente. Publicó en 1690 un libro especializado, ilustrado con imágenes de la extinción de fuegos ${ }^{24}$. En 1721-1725, el londinense Richard Newsham patenta la primera bomba contra incendios accionada por dos hombres, uno a cada lado, subiendo y bajando una palanca. Podía elevar doce litros por segundo de agua a 40 metros de altura (Historia de los bomberos, 2020). Previamente, en París, en 1699, se disponía de 17 aparatos para combatir los incendios y en 1712 la capital francesa contaba ya con 30 bombas, distribuidas en distintos lugares de la ciudad. En Filadelfia, en 1719, apareció el primer vehículo de bomberos

22 «La primera descripción de la historia moderna en máquinas contra incendios se encuentra en una ilustración del libro de Rudolphus Agricola De Re Metalica, publicado en 1556, que presenta, tanto en dibujo como en el taller de un metalúrgico, las distintas piezas de un aparato para combatir el fuego. Cyprian Named Lucarsolase fue el autor de un libro, editado en Londres en 1590, en que se incluía una especie de "jeringa" sobre ruedas, dotada de cilindro y pistón para imprimir presión». Rojo 21 (2015).

${ }^{23}$ Véanse el libro de maquinaria de Heinrich Zeising, en 1612, con la exposición de una bomba de dos cilindros accionada a mano, el libro Forcile Moviments, publicado en 1615, del hugonote francés Caus, el jesuita alemán Gaspar Schott, residente en Konishofen, autor de dos libros de aparatos y máquinas para la extinción de incendios o la monumental bomba «nuremberga» de Hans Hautsch, en 1655; esta máquina consistía en un recipiente circular instalado sobre correderas y un pistón en el centro. Tres hombres accionaban la bomba, además de los que abastecían de agua a la máquina y el que lanzaba agua al fuego, a través del «pistero». El agua salía a presión por la fuerza ejercida en las palancas del pistón, fijas a una pieza horizontal -. Rojo 21 (2015).

24 Véase Rojo 21 (2015) e Historia de los bomberos, CurioSfera (2020). 
con bomba manual (Bomberos en el siglo XVIII, 2019). En Estados Unidos, en 1743, Thomas Lote construyó el primer vehículo destinado al uso de los bomberos con la particularidad de que ya no era tirado por hombres sino por caballos (La evolución de los carros de bomberos en EE. UU., 2020).

Benjamin Franklin, en 1733, tras un viaje a Boston, su ciudad natal, comprobó que sus habitantes estaban mucho mejor preparados para luchar contra los incendios que los residentes en su ciudad de adopción, Filadelfia. Alarmado, inició campañas de prensa y de concienciación sobre la necesidad desesperada que tenían las ciudades de mejorar las técnicas de lucha contra los incendios. El 7 de diciembre de 1736, bajo su influjo, se conformó la Unión de Bomberos. Su equipamiento incluía "cubos de cuero, con las bolsas de fuerte y cestas (para el embalaje y el transporte de mercancías), que iban a ser llevados a cada incendio» (Riquelme González, 2011).

En la España del siglo XVIII, en el Bilbao de 1708 y 1709 se efectúan escrituras de obligación con maestros ensambladores, carpinteros, albañiles y canteros que se comprometían «a acudir a todos los incendios que acaeciesen en la Villa». En 1726 se registra la compra de una nueva bomba de incendios (Guiard Larrauri, 1905). En Santiago de Compostela, en 1784, se pidió, como era preceptivo, al Consejo de Castilla permiso para pagar, de los fondos de Propios y Arbitrios, dos bombas para sofocar los frecuentes incendios (Cebreiros Álvarez, 1997, p. 572). En Soria, a principios de la última década, sobre 1791, la Sociedad Económica de Amigos del País adquiere para la ciudad dos bombas de incendios, valorado el desembolso en 9992 reales (Sanz Yagüe, 2012, pp. 299-301; Rupérez Almajano, 1987, pp. 150-179). En Valladolid, «la primera bomba para combatir los incendios llegó en el siglo XVIII» (Guadilla, 2020). En Madrid, en 1773, se disponía de media docena de bombas, si bien eran consideradas poco prácticas ${ }^{25}$.

En Burgos, en suma, el incendio de la calle La Puebla acució a los responsables del Concejo «con la mira de cortar con más facilidad los Yncendios que ocurran en los Edificios desta Ciudad (...) [se efectuara] la Compra de una Bomba, teniendo presente las proporciones que hay en ella, por las Esguebas que Contiene en su Ynterior, de surtirla con habundancia de Agua $»^{26}$. En febrero de 1769, las Actas de Gobierno recogen la Carta Orden, del 11 de mayo de 1768, expedida por el Supremo Consejo de Castilla «para la compra de una Bomba que corte los yncendios que Ocurran y todos los peltrechos de Picar, Palas y otros, necesarios para el mismo fin (...) sin exceder de los trece mill $r^{5} »$. Era imprescindible, una vez adquiridos, colocar

${ }^{25}$ Vidal Rivas, 2012, pp. 118, 326 y 342-343. En diciembre de 1767 fue contratado por el Ayuntamiento un maquinista hidráulico y constructor de bombas, Juan Jorge Granbner, a quien se concedió, por primera vez, el cargo de "maestro bombero" en Madrid. Véase Cuerpo de bomberos. Maestros Bomberos 1767, 2020 y Granbner, 1773.

${ }^{26}$ AMB. AG. RO del 21 de abril de 1768, folio 140r. 
«estos ynstrumentos en parte segura, cuidándose de su conservaz ${ }^{\text {on }}$, para que pueda servir en los Casos ocurrentes ${ }^{27}$. Se tenía urgencia en la intención de "precaber las Ruinas y estragos que Causan los incendios que, con frecuencia, se experimentan en ella» y que

se remedien los daños qe se orijinan de ellos, sin dar lugar a que los Albañiles hagan higual o mayor sacrificio qe el mismo fuego, porqe con título de Socorrer semejante aflicion cortan y hienden a su Satisfaccion, como les parece y acomoda, y muchas veces sin necesidad de tanto rompimiento ${ }^{28}$.

En el Concejo se tenía noticia de que en la Villa de Bilbao «había una Bomba destinada para estos funestos lances». Era preciso averiguar «si habría Maestro en ella qe la supiera construir y a quanto ascendería su Costo». La respuesta llegó con rapidez: «el costo de la expresada Bomba son nuebe mil Rs $V n$, inclusos materiales y manufactura». Los dirigentes del Ayuntamiento señalaron que «no parece excesiba su estimacion, maiormente siendo una Alaja tan util como necesaria» ${ }^{29}$.

Los responsables municipales entendían que

no sería extraño $q^{e}$ contribuieren todos gustosos con algún voluntario Donatibo, de suerte que por este medio fuese más Suabe, y de menos Costa a V. J., su Conducción, pero sin necesidad de tocar las Puertas del Pobre oficial, se podía facilitar con los Dueños de las Casas que ofrezcan alguna Ayuda de Costa a proporción de sus fuerzas ${ }^{30}$.

Con un pragmatismo cínico, el Concejo planteaba que la Bomba tenía como principal cometido, y «para qe el Común disfrute de Ynstrumento tan util, y si necesita alguna para su perpetuidad pues desde luego se verifica incendio», la extinción de los fuegos urbanos, pero también se podía utilizar «para regar Plazas, Calles y Paseos ${ }^{31}$.

27 AMB. AG. RO del 13 de febrero de 1769, folios 46v-47r. La Carta Orden fue rubricada por Manuel Becerra, Contador General de Propios y Arbitrios del Reino, en Madrid, el 26 de enero de 1769.

${ }^{28}$ AMB. AG. RO del 18 de julio de 1772, folios 251r-251v.

${ }^{29}$ AMB. AG. RO del 18 de julio de 1772, folio 251v.

30 "dándose principio por el Cabildo maior desta Santa Yglesia, sus racioneros, Cabildos y Comunidades Eclesiásticas y regulares de ambos sexos, Maiorazgos y demás Personas particulares que tengan Casas propias en esta Ciudad, siguiéndose después por los demás Vecinos de alguna Sustancia, pues no presume $q^{e}$ hombre alguno de razón se oponga ni resista a contribuir en el modo posible". AMB. AG. RO del 18 de julio de 1772, folio 251r.

${ }^{31}$ AMB. Actas de Abastos (AA). RO del 18 de julio de 1772, folio 252r. De hecho, el encargado de la bomba será, simultáneamente, maestro de obras, alarife, fontanero y "bombero", como se planteará en breve. 
Concedidos los permisos para la compra de la Bomba, se activó el proceso de llevar a cabo «su ejecucion y Conduccion a esta Capital [desde Bilbao] con todos los Peltrechos necesarios, haciendo para ello los Combenios que contemple más beneficiosos al público». Se preveía que se tomara una «completa Ynstruccion, del modo que se debe manejar esta Máquina así para apagar los incendios como para el Regar la Plaza y demás sitios $q^{e}$ se ofrezcan ${ }^{32}$. El desembolso total ascendió a 9000 reales de vellón, con la peculiaridad de que se le había añadido «una Manga de Olona con pintura roja, de quince a diez y seis pies de largo [4,2 a 4,48 metros] de largo, poco más o menos, y su aspa correspondiente para $q^{e}$ por ella reciba el Agua, sin impedir a los operantes q ${ }^{\mathrm{e}}$ le sirba de Gov ${ }^{\mathrm{n}}$ ». Además, se indica que se había sabido «por uno de los que la han manejado y Armado» era necesario <para refrescarla a tiempos porque no se reseque, tubo por conbeniente añadirla el Cañón como seis Baras [4,98 metros] más en lo largo». La bomba debía ser mantenida por esmero porque existía un "defecto», a saber, "el tener la Costura de la Baqueta algo largas las Puntadas, perdiendo por ellas mucha fuerza el Biento». Se advertía que se previniera que «el Artífice diese más menudas las Puntadas» ${ }^{33}$. De hecho, días después se planteaban, de urgencia, otras problemáticas sobre su traslado y conservación ${ }^{34}$.

Los responsables municipales enfatizaban que

no hay Mro que cosa las Mangas de la Bomba de modo que no traspire el Ayre, pues por los Poros de él Corre, traspira y que será por tanta Junta y costura como necesita en tantas piezas a no hacerlas de Metal, no ha nacido ni nacerá quien lo ebite, pero de qe se coserán bien puede em descuidar". Además, "En quanto al Cajón forrado en oja de Lata para custodia de dhas Mangas es superfluo y nocibo $q^{e}$ por haverlas yo encontrado en él las de esta noble villa estaban todas podridas y rotas en los codos y dobles $q^{e}$ hacían dentro de él, por lo $q^{e}$ fue preciso hacerlas nuebas, y después acá no se usa dho Cajón sino en estante tirado alrededor de un quarto, con su Caida correspondiente para $q^{e}$ destilen el Agua después $q^{e}$ Operan se sequen y Oreen en el mismo sitio, se limpian, enseban y gobiernan, pues están a la vista y no en el Cajón, que nadie se acuerda de ellas. El Cajón de madera qe se hará para su conducción serbirá para transportarlas, añadiéndole en esa dos Palos como una Argarilla ${ }^{35}$.

La bomba llegó desde Bilbao. Salvador de Baquijano cobró los 9000 reales, con la salvedad de que, en realidad, la bomba "para extinguir el fuego» había sido, en principio, adquirida para la ciudad de Santander, aunque, finalmente, acabó en Burgos. Se indica que «No binieron Separadamente más Peltrechos ni Piezas q $\mathrm{q}^{\mathrm{e}}$ las

32 AMB. AA. RO del 23 de julio de 1772, folio 254v.

${ }^{33}$ AMB. AA. RO del 13 de agosto de 1772, folios 272v-273v.

${ }^{34}$ AMB. AA. RO del 27 de agosto de 1772, folios 285r-286r.

${ }^{35}$ AMB. AA. RO del 27 de agosto de 1772, folio 285r-286r. 
$q^{e}$ forman dicha Bomba, la que se maneja en su Carrete (...) [y] Todo el Cañón de la Bomba está en tres piezas con sus Tornillos o rocas para el enlace y tan ancho quasi al principio como al medio y fin de cabida o hueco, con el grandor de un Huebo de buen Tamaño» ${ }^{36}$.

En diciembre de 1772, Salvador de Baquijano participaba al Concejo de Burgos que la bomba estaba casi totalmente operativa, aunque, dado que le había dado una última mano de pintura, «necesita algunos días pa Secarse». «Las mangas también están Cosidas, y sólo en el inter se Saca me dibertiré en poner los Tornillos». A principios de enero de 1773, Burgos podría transportarla, para lo que se necesitaba "Un Carromato de dos mulas, por su mucho bolumen, así de la Bomba como del Cajón de las mangas» -su peso era significativo, que «será como Siete a ocho Quintales de a quatro @» $»^{37}-$.

A partir de ese momento, las problemáticas sobre cómo mantener y custodiar la bomba para que fuera lo más útil posible se amontonan. Por una parte, la «Conserbacion» del pertrecho pasaba, necesariamente, por disponer de un espacio en el que ubicarlo. Rafael Rodríguez, su primer mantenedor, indicó que «estaba Colocada en la Cochera qe llebaba a renta el Señor Deán como sitio más a propósito para ello» ${ }^{38}$. Mecanismo de seguridad esencial era el quién iba a disponer de "las llabes (...) para la referida Cochera» ${ }^{39}$. Unos días después, se define que

las llabes de la oficina en que se halla la Bomba se distribuian de este modo: Una al Señor Yntendente Correjidor; otra al Señor Alcalde maior; dos a los Caballeros obreros maiores; una al Señor Diputado del común más antiguo; dos a los Señores Prores maiores Personeros y otra al Ess ${ }^{\text {no }}$ de Ayuntamiento, para que en los casos que ocurran de incendios con promptitud se acuda a sacar esta Machina ${ }^{40}$.

En lo sucesivo la problemática esencial de cuidar de la bomba conocerá una «montaña rusa» de gastos -Gráfico II- en la que lo habitual era que de los Propios se pagara "el trabajo que ha tenido hasta el presente el cuidado de la Bomba» ${ }^{41}$, aunque hubo años en que los desembolsos fueron bastante crecidos. A ello hay que añadir la «fatiga que tubo para apagar» los distintos incendios, razón por la que se

${ }^{36}$ AMB. AA. RO del 13 de agosto de 1772, folio 273rv.

37 AMB. AA. RO del 25 de diciembre de 1772, folio 395r.

${ }^{38}$ AMB. AA. RO del 25 de febrero de 1773, folio 50v. La cochera se ubicaba en el Mercado Mayor.

${ }^{39}$ AMB. AA. RO del 25 de febrero de 1773 , folio $50 \mathrm{v}$.

${ }^{40}$ AMB. AA. RO del 6 de mayo de 1773, folio 98v. Véase Sanz Yagüe, A. I., 2018.

${ }^{41}$ Véase, por ejemplo, de entre todos los posibles, AMB. Actas de la Junta de Propios y Arbitrios (JPA). RO del 4 de septiembre de 1773, folio 104r. 
GRÁFICO II. GASTOS EN MANTENIMIENTO DE LA BOMBA

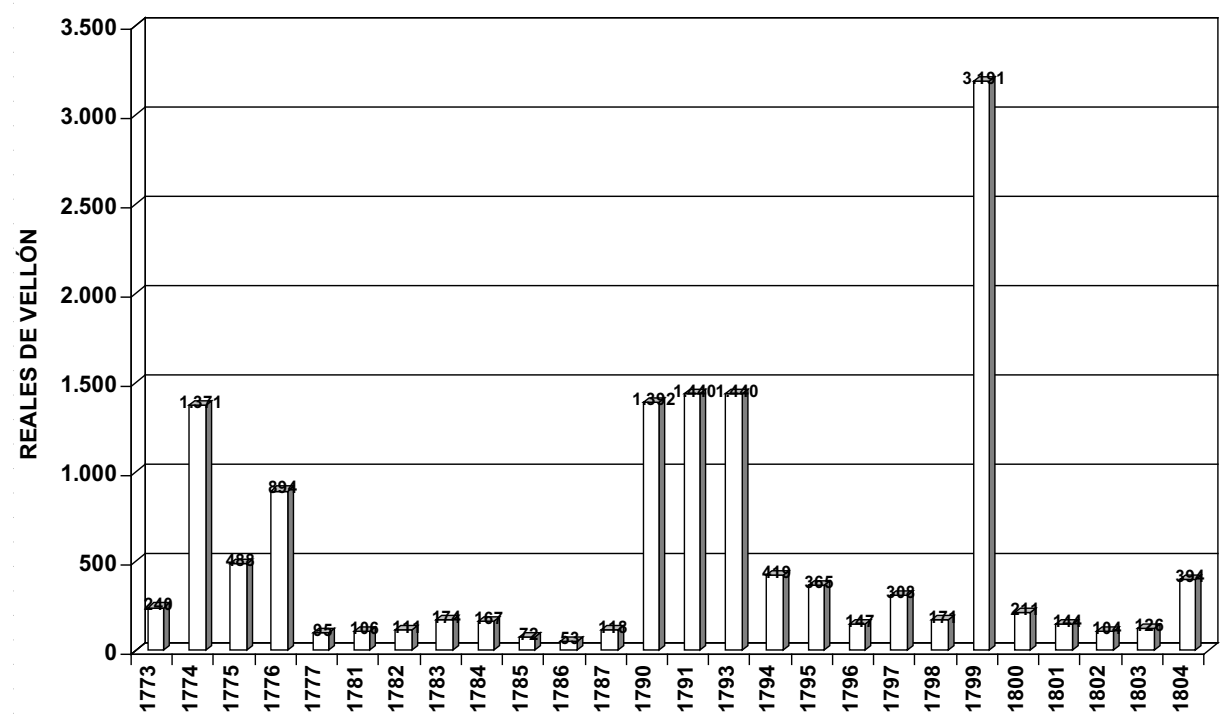

Fuente documental: AMB. AG, AA y JPA. Múltiples años (1700-1799).

demandaba una "gratificación» especial ${ }^{42}$. Su cometido principal, además de acudir a la extinción de los incendios en los momentos excepcionales, era, en lo habitual, «abilitar la Bomba y la Untura de sus Mangas» ${ }^{43}$.

No hemos de olvidar, en todo caso, que, como se observa en el Gráfico III, se produjo, en el Burgos de la segunda mitad del Setecientos, un fenómeno aparentemente contradictorio y sardónico. El Ayuntamiento habría de resignarse a pagar no solo el mantenimiento y conservación de la bomba para apagar los incendios sino, también, las actuaciones para la extinción de las llamas en dichos episodios, al margen de la bomba -como se irá desvelando en las próximas páginas-. Resulta paradójico, e irónico, además de sorprendente en la práctica, que la compra de una bomba para apagar incendios denotara un antes y un después en el número de ocurrencias y, sobre todo, de los montantes a pagar en tales acontecimientos.

Se denota una contradictoria relación entre el número de incendios habidos en la ciudad de Burgos -Gráfico I-, el gasto municipal en el mantenimiento de la bomba - Gráfico II-y los desembolsos efectuados por el erario público en el tratamiento de los dichos incendios -Gráfico III-. Aunque los ritmos zigzaguantes de unos y otros no fueron puntualmente determinantes, en la práctica nos topamos con un devenir creciente en los tres aspectos. Si como se ha dicho en páginas anteriores hasta 1768 -incendio de casa de la calle La Puebla- las ocurrencias fueron escasas

42 AMB. JPA. RO del 4 de septiembre de 1773, folio $104 \mathrm{r}$.

43 AMB. AA. RO del 4 de julio de 1776 , folio $106 \mathrm{v}$. 
GRÁFICO III. GASTOS EN EXTINCIÓN DE INCENDIOS

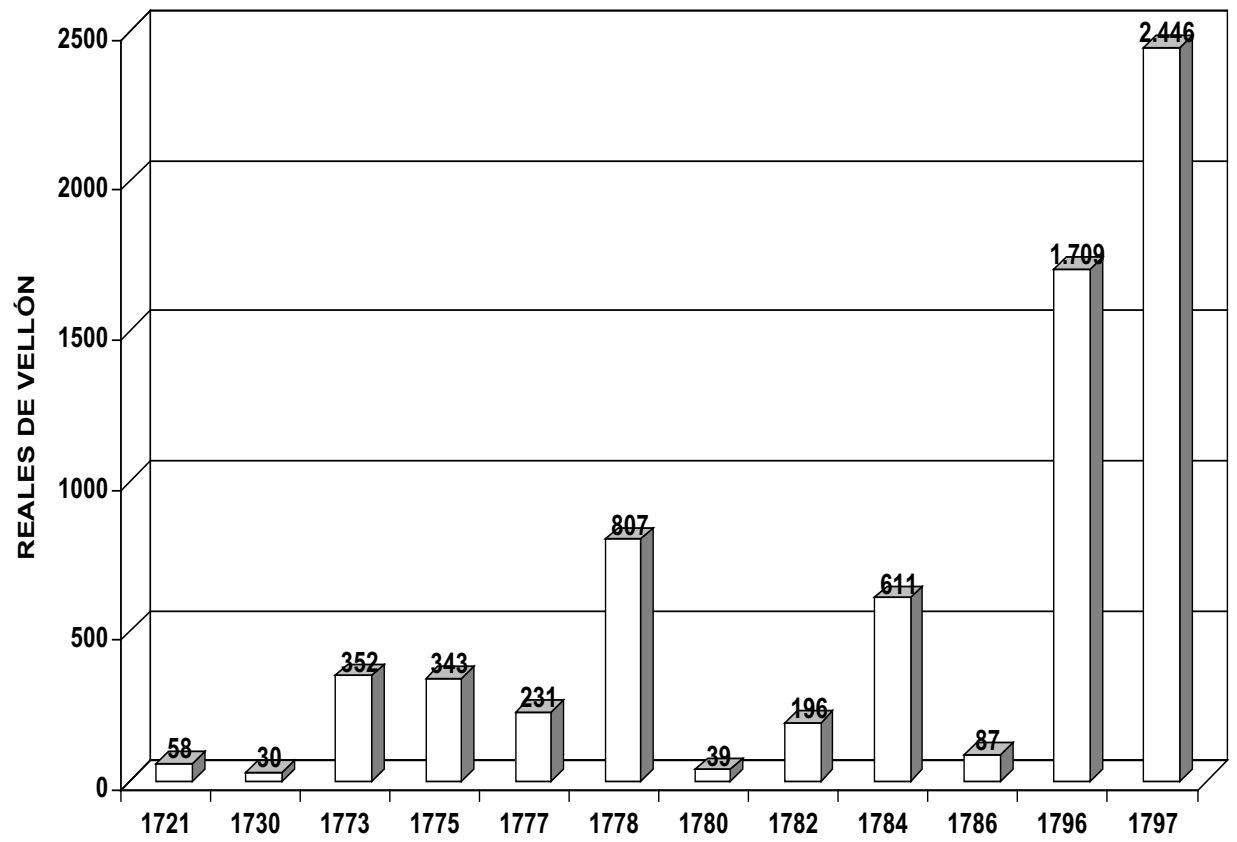

Fuente documental: AMB. AG, AA y JPA. Múltiples años (1700-1799).

y con facturas de reducidas proporciones ${ }^{44}$, desde la adquisición de la bomba, fabricada en Bilbao, los incendios van a más no solo en número sino también en su coste para los Propios, aunque se aprecian momentos especialmente gravosos. En la calle La Calera (1773), 352 reales ${ }^{45}$, en Pozo Seco (1775), 343 1/2 reales $^{46}$, en San Esteban (1777), 231 1/2 reales ${ }^{47}$ y, en especial, el ocurrido en el Colegio de Carmelitas (1778), 807 reales $^{48}$. Después, los incendios fueron, de nuevo, baratos, pero

${ }^{44}$ El incendio en la cárcel (1721) supuso 58 reales -AMB. AG. RO del 17 de febrero de 1721, folio $61 r-y$ el incendio en una casa del Mercado Mayor (1730) únicamente 30 reales- AMB. AG. RO del 2 de diciembre de 1730, folio 298v-.

45 AMB. JPA. RO del 4 de septiembre de 1773, folios 108v-109r, AA. RO del 23 de septiembre de 1773 , folios $206 v-207 r$, JPA. RO del 24 de septiembre de 1773, folios 108v-109r y JPA. RO del 14 de octubre de 1773 , folios $126 \mathrm{v}-127 \mathrm{v}$.

${ }^{46}$ AMB. AA. RO del 10 de marzo de 1775 , folios 60r-61r.

47 AMB. AA. RO del 4 de diciembre de 1777, folios 240v-241v.

${ }^{48}$ AMB. AA. RO del 5 de febrero de 1778, folios 30v-31r y JPA. RO del 12 de febrero de 1778, folio 43rv. 
en crecimiento sostenido. Véanse, en 1780, el de calle San Cosme, $391 / 2$ reales $^{49}$, el de la calle San Juan (1782), $1961 / 2$ reales $^{50}$, los 611 1 $/ 2$ reales de tres casas en Santa Águeda $(1784)^{51}$ y los $871 / 2$ reales del cuartel de Artillería (1786) ${ }^{52}$. Tales desembolsos fueron bagatelas si los contrastamos con el acaecido en las casas de los Alcaldes Mayores, en la Plaza Mayor (1796) ${ }^{53}$ y la enorme destrucción y los considerables gastos del incendio en las casillas del Mercado Mayor (1797), 2446 reales $^{54}$. Tales facturas se añadían a las derivadas del mantenimiento de la bomba y sus pertrechos -especialmente gravosas en $1790-1793$ y 1799-.

Empero, a pesar de todos los problemas asociados a la bomba y a los incendios, el que fuera Corregidor e Intendente de Burgos (1765-1776), Miguel Bañuelos, a la sazón intendente de los Ejércitos en el Reino de Galicia en 1785, solicitó del Concejo de Burgos - no olvidemos que bajo se mandato en la capital castellana se adquirió la bomba para cortar "los frecuentes incendios de que estaba aflixida esa honrada Poblacion y tenían en continuo desasosiego al Gobierno» ${ }^{55}$ - que se le remitiera

un planito de dha bomba con sus mangas, El carro embetunado que ahí se le añadió por discreta prevención para surtirla incesantemente de agua y explicación de los cubos de Cuero, escaleras, maromas y demás Utensilios de que se la probeyeron para usarla oportunam ${ }^{\text {te }}$, como así mismo del Maestro que la hizo y el coste que tubo el todo ${ }^{56}$.

\section{ORIGEN DEL “BONBERO» Y SUS COMETIDOS EN EL SIGLO XVIII BURGALÉS}

A lo largo del último tercio del Setecientos, desde la adquisición y llegada a Burgos de la bomba para la extinción de incendios, cuatro individuos fueron los encargados de su mantenimiento. La primera vez que aparece, de forma explícita,

${ }^{49}$ AMB. AA. RO del 9 de marzo de 1780 , folios $44 v-45 r$.

${ }^{50}$ AMB. JPA. RO del 12 de enero de 1782, folio $14 \mathrm{r}$.

${ }^{51}$ AMB. AA. RO del 23 de diciembre de 1784, folio 225rv y JPA. RO del 29 de diciembre de 1784, folios $108 \mathrm{v}-109 \mathrm{r}$.

${ }^{52}$ AMB. AA. RO del 26 de octubre de 1786, folio 193v.

${ }^{53}$ AMB. JPA. RO del 15 de febrero de 1796, folio $2 \mathrm{v}$.

${ }^{54}$ AMB. JPA. RO del 27 de junio de 1797, 18v y AA. RO del 13 de julio de 1797, folios $185 \mathrm{v}-186 \mathrm{r}$.

${ }^{55}$ AMB. AA. RO del 15 de septiembre de 1785, folio 198r. El incendio de la calle La Puebla de abril de 1768 acompañó a Bañuelos durante el resto de su existencia.

${ }^{56}$ AMB. AA. RO del 15 de septiembre de 1785, folios 197v-199r. La carta de Bañuelos en La Coruña el 7 de septiembre de 1785. 
la denominación "Bonbero» (sic) fue el 28 de febrero de 179957. Al fallecer cada uno de ellos, el Ayuntamiento se veía obligado a contratar a otro operario, con las problemáticas que surgían de la competencia entre vecinos por dichos quehaceres. La tendencia fue acumular en una misma persona los empleos de alarife, fontanero y cuidador de la bomba, circunstancia no exenta de conflictos y litigios.

Rafael Rodríguez fue "Director de la Bomba ${ }^{58}$ entre 1773 y $1781^{59}$. Durante ese período, tras el desembolso de los 9000 reales iniciales, se fueron acumulando quehaceres de mantenimiento y mejoras urgentes, derivadas del descubrimiento de disfunciones de la bomba en lo momentos más álgidos ${ }^{60}$. En octubre de 1773 se acometió «hacer las nuebas Ruedas (...) para conseguir con más facilidad el manejo de la Bomba», con un gasto de 240 reales -Gráfico II-61. En 1774, fueron 435 reales ${ }^{62} y$, después, otros 936 reales $^{63}$-en total, 1371 reales-. En 1775, un memorial de Rafael Rodríguez urgía al Ayuntamiento a mejorar el servicio con mayor eficacia. Se solicitaba «Un premio de quatro, tres u dos reales Vellón según y conforme lleguen las Personas para conducirla [se refiere a la bomba], medio que nos muestran las Probincias más bien policiadas de la Europa, con el que han conseguido la mayor satisfacción». Se demandaba que los alcaldes de barrio de las colaciones de San Juan y San Lesmes, «en cuio distrito se conserba esta preciosa [e] importante Máquina, sean los primeros que asistan a auxiliar al Director de ella» y entregan a las personas implicadas en sofocar los incendios <los Baldes, Cubetos, Palas y Azadones y

57 AMB. AA. RO del 28 de febrero de 1799 , folio $50 \mathrm{v}$. «Llamam ${ }^{\text {to }}$ sobre si se han de proveher juntos o separados los encargos de Alarife, Fontanero o Bonbero".

${ }^{58}$ AMB. AA. RO del 7 de febrero de 1775 , folio 10rv.

59 El 5 de octubre de 1781 se informa a los responsables del Concejo de la defunción de la «persona que cuidaba de la Bomba que sirbe para los incendios que acaezen en esta Ciudad y haber quedado bancante dho empleo". AMB. AG. RO del 5 de octubre de 1781 , folio $219 \mathrm{v}$. Por el cuidado de la bomba, Rafael Rodríguez disfrutó de un salario diario de 1 real. Véase, por ejemplo, AMB. JPA. RO del 29 de octubre de 1774, folio $161 \mathrm{v}$.

60 Las autoridades municipales eran conscientes de que era imprescindible «que la Ciudad tenga un repuesto de instrumentos de gastador, Cuerdas, Escaleras y otros útiles que auxilien a la Bomba para cortar y apagar los fuegos». En concreto, se precisaba «aumentar los Cubos de Cuero, Calculando su importe (...) pues sin estos agregados será la Bomba de menos aprovechamiento". AMB. AA. RO del 2 de septiembre de 1773, 190v. Situaciones similares se vivían en otras ciudades. Así, por ejemplo, en Chartres, donde «les pompiers de la ville et leur matériel ("pompes, sceaux, échelles, pelles, piques et pioches») se movilizaban en los incendios, además de que «sont vite assistés de plusiers dizaines d'habitants». Garnot, 1989, pp. 206-207.

${ }^{61}$ La «Quenta deel Costo de quatro ruedas» supuso un desembolso de 240 reales. AMB. AA. RO del 25 de octubre de 1773 , folio $264 \mathrm{v}$.

62 AMB. JPA. RO del 18 de febrero de 1774 , folio 35 r. Se compraron hachas y picos y se reconocieron las mangas de la bomba.

${ }^{63}$ AMB. JPA. RO del 29 de octubre de 1774, folios 159v-160r. Se adquirieron maromas en Nájera, hachas, picos, cubos,... 
demás útiles», procurando después "recoger quantos Pertrechos ban mencionados y entregárselos al Director de la Bomba», quien los conducirá "a su sitio sin la menor abería». Rodríguez exigía, también, mayor cantidad de cántaros, repartidos por todas las collaciones, «quatro Faroles de Talco de mano y ocho con sus Baras para fijarlos en el distrito y Calle donde acaezca el Yncendio y las Belas correspondientes para ellos», "dos Bocinas, que una ha de serbir para el Director de la Bomba» y una escalera de proporcionada altura ${ }^{64}$. Todo ello supuso un gasto de 488 reales $^{65}$.

En 1776, el mantenimiento de la bomba obligó a gastar $894 \frac{1}{2}$ reales ${ }^{66}$. Posteriormente, fueron más leves ${ }^{67}$. En septiembre de 1780 , se precisó que «un Mro de su satisfaccion repare la ruina que tubiere el Almazen en que se custodian la Bomba y sus peltrechos ${ }^{68}$ y en 1781 mantecas y grasa "para untar la Bomba» y sacarla a remojar múltiples veces (1779-1780) 106 reales y 24 maravediés ${ }^{69}$-Gráfico II-.

Fallecido Rafael Rodríguez, su hijo Tomás se ocupó en el «cuidado y conserbacion de la Bomba» en 1781-1786. Los ediles acordaron que se le hiciera saber "a el que cuida de la bomba y sus Peltrechos cumpla con las obligaciones que tiene contraidas para su conservacion sin faltar en cosa alguna, con apercibimiento de que en su defecto será pribado de este encargo ${ }^{70}$. Tomás Rodríguez gastó, en 1783, 111 reales en el cuidado del artefacto -refresco, velas, faroles y tornillos ${ }^{71}$ y en 1783174 reales $^{72}$. En el Concejo se detectan serias dudas sobre la profesionalidad del susodicho Tomás. De hecho, se trajo a colación la «Receta del Maestro constructor de la Bomba para su conserbazion» como llamada de atención al cuidador «a fin de que execute la untura o compuesto que refiere para la conserbacion de

${ }^{64}$ La escalera serviría «para subir por ella el Director de la Bomba, pues se be muchas veces que ni por la Casa incendiada o por las contiguas a ella, o por ser Casa sola la desgraciada, se puede manejar el Cañón de la Bomba, y deste modo, o por las Ventanas o haciendo algún ahujero en los Tabiques exteriores se logra el fin de apagar el incendio». AMB. AA. RO del 7 de febrero de 1775 , folios 10r-12v.

${ }^{65}$ AMB. AA. RO del 16 de marzo de 1775 , folios 66v-67r,

${ }^{66}$ En cuatro partidas de105 reales, 132 reales, 216 reales y 300 reales en febrero -AMB. AA. RO del 29 de febrero de 1776, folios $41 v-43-$ y $141 \frac{1}{2}$ reales en julio -AMB. AA. RO del 4 de julio de 1776 , folio $106 v-$.

6736 reales en febrero de 1777 -AMB. AA. RO del 3 de febrero de 1777, folio 39 r- y 59 reales en diciembre-AMB. AA. RO del 18 de diciembre de 1777, folio 250rv-.

${ }^{68}$ AMB. AA. RO del 15 de septiembre de 1780 , folio $239 \mathrm{v}$.

${ }^{69}$ AMB. AA. RO del 12 de febrero de 1781, folios 44v-45r.

70 Como se comprobará posteriormente, existían serias dudas sobre la fiabilidad de los quehaceres de los nuevos directores de la bomba. AMB. AG. RO del 10 de junio de 1782, folio $167 v$.

${ }^{71}$ AMB. AA. RO del 9 de enero de 1783, 3v.

72 "gasto causado en su conserbacion y limpieza». AMB. AA. RO del 15 de enero de 1784, folio 18 r. 
las Mangas»" ${ }^{73}$. Los "gastos causados en la preparacion de poner sensible la Bomba y todos sus utiles correspondientes y nezesarios» en 1764 ascendieron a 167 1/2 reales $^{74}$.

Con el óbito del alarife Julián de Arbaiza ${ }^{75}$ se planteó en el Concejo la unificación en una solo persona de los salarios y quehaceres de alarife, fontanero y cuidador de la bomba -2140 reales- ${ }^{76}$. Era habitual en semejantes procesos la posibilidad de que hubiese como "Pretendte algun Maestro o Maestros idoneos para serlo [y] que se encargase del ministerio de fontanero y cuidado de la Bomba ${ }^{77}$. Se presentaron ante el Concejo memoriales de Francisco Céspedes ${ }^{78}$, quien se hizo con tales menesteres (1786-1798) ${ }^{79}$. No obstante, existió una disociación funcional, dado que Tomás Ángel Rodríguez, vecino también de Burgos, se ocupaba de «sacar a refrescar la Bomba», que en 1786 ascendió a 53 reales $^{80}$-Gráfico II-. Las insinuaciones de los responsables municipales para que se tomaran, por parte de Francisco Céspedes, «quantos medios y disposiciones combengan a tenerla corriente para qualquiera acontecimiento», delate una cierta disintonía entre el obligado de la bomba y la persona que, cotidianamente, se habría de encargar de su cuidado -si Céspedes se ausentaba de la ciudad o sufría algún accidente "quede Suxeto que con conocimiento y utilidad la pueda usar ${ }^{81}-$. En 1788 , los gastos de mantenimien-

${ }^{73}$ Baquijano, el bilbaíno constructor de la bomba, había remitido al Ayuntamiento una "Receta con su dosis de cada cosa para untar las mangas de la Bomba. Manteca de puerco sin sal doze libras. Las ezes de Grasa seis libras. Alquitrán fino tres libras. Todos mixtos, se derretirán a fuego manso a que se incorporen bien, y como dho es se usará estos condimentos, sea en mayor cantidad o menor, se proporcionaran cada cosa». AMB. AA. RO del 6 de febrero de 1784, folio $38 \mathrm{v}$.

${ }^{74}$ AMB. JPA. RO del 19 de marzo de 1785, folios 43v-44r. En 1785 se gastaron 72 reales AMB. JPA. RO del 14 de enero de 1786, folio 11 r-y en 178653 1/2 reales -AMB. AA. RO del 7 de diciembre de 1786 , folio $211 \mathrm{v}$-.

75 Iglesias Rouco, 1978, pp. 134. Arbaiza era maestro de obras y alarife de la ciudad y maestro de obras del Cabildo de la Catedral. Vivía en la bajada a Barrantes, donde su cónyuge gestionaba una posada para estudiantes. Véase también pp. 117, en lo tocante a la bomba de incendios.

${ }^{76}$ AMB. AG. RO del 17 de julio de 1786, folio 136rv.

77 AMB. AG. RO del 14 de agosto de 1786, folios 148v-149r.

${ }^{78}$ AMB. AG. RO del 4 de septiembre de 1786, folio 163rv y AG. RO del 11 de septiembre de 1786, folios $182 \mathrm{v}-184 \mathrm{r}$.

79 AMB. AG. RO del 11 de septiembre de 1786, folios $182 v-184$ r y AG. RO 5 de octubre de 1786, folio 204rv. Francisco Céspedes percibiría 112 1/2 reales «por tener corrientes las Fuentes de la Ciudad» y por el empleo de alarife y fontanero 1500 reales/año, a lo que se añadía el real/ día por "el cuidado de la Bomba».

80 AMB. AA. RO del 7 de diciembre de 1786, folio 211v.

${ }^{81}$ AMB. AA. RO del 13 de septiembre de 1787, folio $196 \mathrm{v}$. 
to de la bomba supusieron 118 reales $^{82}$. Fuera por negligencias en su cuidado o por la obvia obsolescencia de los materiales, en 1790-1793 las inversiones municipales en la bomba se dispararon, y mucho. En 1790, "los costos causados para reponer los utiles de la Bomba, ochocientos caños comprados para las Fuentes» importaron 1382 1/2 reales $^{83}$, en 17931440 1/2 reales $^{84}$-Gráfico II-. Francisco Céspedes, en 1792, expuso ante los componentes del Consejo, en un memorial,

como algunos Cañones de suela se hallan deteriorados, y $\mathrm{p}^{\mathrm{a}}$ que en caso de que alguno de ellos falte en algunas de las operaciones, benía bien que se tubiesen otros de repuesto, pues de no haverlo, como están tan manidas las actuales, se podría quedar inservible ${ }^{85}$.

Los siguientes años (1795-1797) los gastos en el mantenimiento de la bomba no fueron disparatados ${ }^{86}$ pero se dispararon las alarmas. En marzo de 1795, al hilo del incendio que se desató en el convento de San Pablo

se havia experimentado con dolor que deviendo estar la Bomba corriente para atajarle no havia producido efecto alguno y tomado más incremento $q^{\mathrm{e}}$ el $\mathrm{q}^{\mathrm{e}}$ podía esperarse, sin duda $\mathrm{p}^{\mathrm{r}}$ poco cuidado y mala administraz ${ }^{\text {on }}$ del Alarife fran ${ }^{\mathrm{c}}$ Cespedes, a quien está encargada y percive sueldo por ello ${ }^{87}$.

Los ediles achacaban a Céspedes que se había roto «el expediente o conducto

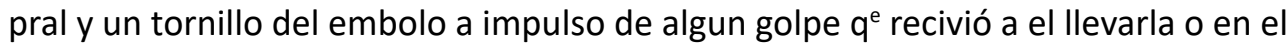
mismo Sitio a el armarla, qual se experimentó por la tarde». Sin titubeos, acusaban al alarife y fontanero de culpable de negligencia, "pues luego $q^{\mathrm{e}}$ se puso dho tornillo se vió corriente y en disposición de hacer toda el agua $q^{e}$ se quisiese" ${ }^{\prime 88}$. Céspedes se consideraba inocente y afirmaba, «como se puede justificar por vastantes del Pueblo», "estuvo todo el Dia refrescandose la máquina y Cubos de suela y los Cántaros dos días». Si había fallado podría ser el resultado de averla tenido "sin uso desde

82 AMB. JPA. RO del 28 de marzo de 1788, folios 25v-26r.

83 AMB. AA. RO del 11 de marzo de 1790, folios 65v-66r.

${ }^{84}$ AMB. AA. RO del 19 de enero de 1792, folio 38v y JPA. RO del 9 de febrero de 1793 , folio $5 v$.

85 AMB. AA. RO del 19 de enero de 1792, folio 38v.

${ }^{86}$ En 1795 ascendieron a 419 1/2 reales-AMB. AA. RO del 6 de febrero de 1794 y AA. RO del 9 de abril de 1795, folio 127r-. En 1796, 147 reales -AMB. AA. RO del 12 de febrero de 1796, folios 47v-48r-y en 1797308 reales -AMB. AA. RO del 16 de febrero de 1797, folio 49v-.

87 AMB. AG. RO del 9 de marzo de 1795, folio 106rv.

${ }^{88}$ AMB. AG. RO del 9 de abril de 1795, folio $126 \mathrm{v}$. 
aquel tiempo hasta el de la Casualidad». Los «Quebrantos» de que le acusaban los regidores derivaban de que las piezas de la bomba

se pierden por mojarse tanto, pues su firmeza solo consiste en un Ylo de Cañamo y este con la Umedad se pudre, vien $q^{e}$ yo estoi obligado a cumplir con los preceptos y la Sacaré quantas Veces se me mande, pero lo cierto es $q^{e}$ el $q^{e}$ la cuida en Bilbao me dixo $q^{\text {e }}$ lo q e convenía para su durazion era el untarla todos los meses y así lo ha hecho". En resumen, no existía negligencia de su parte ni "intervino culpa ni aún descuido en el citado acontecimiento ${ }^{89}$.

Después de que se trató y "conferenciandose $\operatorname{largam}^{\text {te }}$ », se acordó, por el mayor número de votos, que se quitaran los 365 reales del salario por cuidar la bomba y que «la falta de la Bomba en el incendio de San Pablo consistió en el rompimiento del emvolo y expediente, causado de un golpe a el llevarla». Era preciso reparar las averías y remojar los cañones y los cubos más a menudo ${ }^{90}$. Se decidió igualmente escribir a Bilbao y pedir sugerencias y ayuda. La respuesta no se hizo esperar y se dijo lo siguiente:

el Govierno de las Bombas y mangas se reduce así: en el Cuerpo de la máquina o en su interior sino hay descalabrada en alguna Balbula será necesario soltarla y registrarla o sino podrán estar los embolos o chupones resecados y si están así, sacandolos dhos embolos por los Cañones, se umefecerán o ajustarán en los Cañones con cuidados suavizados con manteca sin sal, vien untados, colocándolos en su lugar con cuidado. Las mangas de suela si están resecadas, de no haver tenido cuidado de untarlas a menudo con manteca sin sal, estarán duras y de ningún servicio, y la costura estará podrida, y para estos es necesario soltar las dhas mangas con cuidado, suavizarlas y bolverlas a coser con cuidado, $q^{e}$ quedarán como nuevas ${ }^{91}$.

La inactividad de la bomba era un grave problema para su persistencia. En octubre de 1796, los procuradores mayores propusieron que «con ocasión de haver asistido a la inutilizacion del trigo de mala calidad» se había llevado la bomba "para hechar agua en las Zanjas», circunstancias que les llevó a advertir que «sin duda por lo reseca de los cañones de vaqueta está por largo tiempo inútil». En efecto, habían comprobado que «si de tiempos en tiempos no se remoja, haciendo uso de ella, se verificará $q^{e}$ en el mayor apuro no surtirá los prontos efectos $q^{e}$ se desean de apagar los incendios q pueden ocurrir». En la práctica, era una denuncia de que Céspedes estaba obligado al «cuidado de dha Bomba (...) tenerla vien acondicionada, untados

\footnotetext{
89 AMB. AA. RO del 23 de abril de 1795, folio 139ar.

${ }^{90}$ AMB. AA. RO del 23 de abril de 1795, folio 139v-140r.

${ }^{91}$ AMB. AA. RO del 21 de mayo de 1795, folios 171v-172r.
} 
los cañones» y sacada y usada "cada quince Días ${ }^{92}$. Y no había excusas. Como se demuestra en el Gráfico III los incendios de 1796 y 1797 fueron auténticamente pavorosos y destructivos, en especial los acaecidos en la Plaza Mayor y el Mercado Mayor. A más inversiones en bomba más llamas en las propiedades urbanas del Ayuntamiento.

Los gastos causados «en el refresco y untura de la Bomba $q^{\mathrm{e}}$ sirve para los incendios» importaron, en 1798, 171 1/2 reales $^{93}$. Empero, los regidores consideraron que «la Bomba se halla abandonada, mal administrada e inservible para qualq ${ }^{a}$ ocurrencia de fuego $\mathrm{q}^{\mathrm{e}}$ se experimente, sin duda por desidia del Mro Alarife $q^{\mathrm{e}}$ está encargado de su cuidado y custodia $»^{94}$. La «dolorosa ocurrencia del fuego del combento de la Victoria» " impulsó, entre los ediles, la adopción del "arreglo de un método pa las ocurrencias del fuego" y, a la postre, la propuesta de buscar un nuevo alarife, fontanero y «Cuidador de la Bomba». En la práctica, suponía que, aunque Céspedes llevara 10 años en dichos quehaceres, era apetecible un drástico cambio ${ }^{96}$.

El fallecimiento del susodicho Francisco Céspedes ayudó. Se propusieron para dichos empleos de alarife, dirección de fuentes y cuidado de la bomba los maestros de obras Miguel de Gorrachategui, por una parte, y Tomás Rodríguez, por otra ${ }^{97}$. Los responsables del Ayuntamiento dudaban sobre «si se han de proveer juntos o

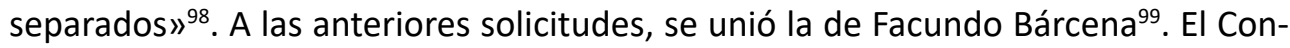
cejo optó por Tomás Rodríguez y por invertir, en 1799, en la recomposición de la bomba para cortar incendios de una cantidad sobresaliente, 3143 reales $^{100}$-Gráfico II-. Tomás Rodríguez procuraba tener la bomba «en el estado más corriente para ocurrir a qualquiera impensado incendio», lo que, en 1800 , supuso 211 1/2 reales ${ }^{101}$. El contraste con lo ocurrido en estos menesteres en 1849 nos descubre que, de vez en cuando, se hacía imprescindible sanear la bomba, sobre todo cuando se advertía de su «malísimo estado, ya por su antigua y mediana Construcción o ya quizá por descuydo». Las mangas, a la larga, se pudrían, era preciso reponerlas y ello

92 AMB. AA. RO del 27 de octubre de 1796, folio 348r.

93 AMB. AA. RO del 19 de abril de 1798, 105v-106r.

${ }^{94}$ AMB. AA. RO del 11 de julio de 1798, folio 204r.

95 Incendio fechado el 19 de agosto de 1798. AMB. AA. RO del 23 de agosto de 1798, folios 231r-232v.

96 AMB. AA. RO del 23 de agosto de 1798, folios 235v-236r y AG. RO del 19 de noviembre de 1798 , folio 143 r.

${ }_{97}$ AMB. AA. RO del 18 de noviembre de 1798, folio 325rv. En su memorial al Concejo, Tomás Rodríguez reivindica se le asigne el trabajo como una fórmula para mantener la tradición, dado el «mucho tiempo [que estuvo] a cargo del Padre del suplicante», a pesar de sus enfermedades.

98 AMB. AA. RO del 10 de enero de 1799 , folios $4 v-5 r$.

${ }^{99}$ AMB. AA. RO del 7 de febrero de 1799 , folios $32 v-33 r$.

100 AMB. AA. RO del 20 de junio de 1799, folio $117 \mathrm{v}$.

${ }^{101}$ AMB. AA. RO del 19 de diciembre de 1800, folios 307r-308r. 
incrementaba, de forma notoria, los desembolsos. En 1849, hasta 2.488 reales -con el agravante de que tanto el trabajo como los materiales se encargaron en París, «adbirtiendo $\mathrm{q}^{\mathrm{e}}$ son de la mejor calidad q" se fabrica»- ${ }^{102}$.

\section{A MODO DE CONCLUSIÓN}

La reconstrucción de los procesos de lucha contra los fuegos urbanos y la búsqueda de métodos eficaces para la extinción de las llamas nos demuestra que durante gran parte del siglo XVIII esta faceta se encontraba en pañales en la ciudad de Burgos. Los incendios hasta 1768 fueron pocos y baratos. El Corregidor e Intendente Bañuelos no solo impulsó el «Hermoseamiento» de los edificios de la ciudad, sino que, además, definió una estrategia de combate contra el fuego basada en la operatividad de una bomba diseñada para el rápido despliegue de una maquinaria versátil y «moderna» de lucha contra los incendios en el tejido urbano. El Ayuntamiento se vio envuelto en una espiral de inversiones y gastos paradójica, según la cual se incrementaban, a veces de manera estridente, los desembolsos en la bomba y sus pertrechos y los gastos en cada vez más incendios y cada vez más exigentes en dinero. Un aspecto esencial fue la creación de la figura del "Director de la Bomba» y, a la postre, del "Bonbero», que aunaba en sí las peculiaridades propias de un alarife y maestro de obra con las de fontanero y cuidador de la bomba. Sus actuaciones estuvieron, en ocasiones, marcadas por claros y oscuros, en la medida en que la bomba, y sus herramientas complementarias, estuvieran operativas y no dejaran a la Ciudad en el ridículo de ver arder edificios notorios sin poder hacer absolutamente nada o bastante poco.

Para no sobrecargar en exceso estas páginas se ha quedado en el tintero el seguimiento de algunas circunstancias que, en la práctica, eran vórtices peligrosos, e inflamables, para la salud de las edificaciones burgalesas. Descuellan, en especial, las instalaciones de hornos para fábrica de mantas tintadas en la Plaza Mayor orquestadas por Ildefonso de Corrés Marañón y Foronda (1752-1758), denunciadas por los maestros artesanos y sus vecinos como potencialmente letales para sus viviendas o las problemáticas derivadas del uso de la pólvora -para la generación de armamento o para los fuegos lúcidos en las fiestas-. A ello se añaden los peligros, siempre latentes, aquí y allí, en los hornos de yeso generados en los quehaceres de la construcción urbana, los tinglados de los maestros latoneros para la fundición de metales. $\mathrm{O}$, a la postre, la necesidad de trasladar los mesones a espacios lejanos del centro urbano para evitar incendios por la acumulación de paja, animales y personas en espacios reducidos, hacinados y necesitados de calor, sobre todo en la rigurosidad de los inviernos, dada las frialdades habituales en la ciudad castellana.

102 AMB. Histórica. Legajo 22-360 (13 y 24 de abril, 4 de mayo y 1 de julio de 1849), sin foliar. 


\section{REFERENCIAS BIBLIOGRÁFICAS}

Abbad, F. y Ozanam, D. (1992). Les intendants espagnols du XVIII' siècle. Madrid: Casa de Velázquez.

Abbad, F. (1982). Hacia una historia de la Intendencia en la España Moderna. Cuadernos de Investigación Histórica, 6, pp. 103-108. https://doi.org/10544/ etfiv.30.2017/ 18554.

Anero Cárcamo, M. (2007) Técnicas de investigación de incendios. Incendios de origen eléctrico. Barcelona: Universidad Autónoma de Barcelona.

Belmonte López Huici, M. C., Cuesta Martínez, M., García Cano, M. I. y Pozas Poveda, L. (1987). Las actas capitulares como fuente para la historia urbana. En la España medieval, 10, pp. 39-68. https://doi.org/10.5209/ELEM.

Bomberos en el siglo XVIII. (2019). Grupo TME. Recuperado el 16 de julio de 2020, de https://grupotme.com/bomberos-en-el-siglo-XVIII.

Bravo, A. (2019). Bomberos. Una historia de lucha contra los incendios. Academia Play. Recuperado el 16 de julio de 2020, de https://academiaplay.es/bomberosuna-historia-de-lucha-contra-los-incendios.

Campos y Sáez, C. A. (1984). Técnica y sociedad en los siglos XVII y XVIII. En MHormigón Blánquez (coord.), Actas del II Congreso de la Sociedad Española de Historia de las Ciencias. Jaca: SEHCYT, Vol. 3.

Cebreiros Álvarez, E. (1997). El municipio de Santiago de Compostela a finales del Antiguo Régimen (1759-1812). La Coruña: Universidad de La Coruña.

Coll Coll, A. M. (2015). La Intendencia de Mallorca en el siglo XVIII. Palma de Mallorca: Universitat de Les Illes Balears. https://doi.org/10.5944/etfiv.30.2017.18554.

Cuerpo de bomberos. Maestros Bomberos 1767. (2020). Recuperado el 16 de julio de 2020, de https://www. Madrid.es/portales/munimadrid/es/Bomberos/ Cuerpo-de-Bomberos.

García Ruipérez, M. (1999). El Archivo Municipal de Toledo y la investigación histórica sobre la Edad Moderna. Cuadernos de Historia Moderna, 22, pp. 201-227. 
Garnot, B. (1989). Le logement populaire au XVIII siècle: L'exemple de Chartres. Revue d'histoire moderne et contemporaine, 36, pp. 206-209. https://doi. org/10.3406/rhmc.1989.1490.

Granbner, J. (1773). Explicación de las bombas más necesarias y útiles para qualquier inzendio. Archivo Histórico de la Villa de Madrid, Legajo 1-88-42.

Guadilla, M. A. (2020). Sucedió en Valladolid. El primer cuerpo de bomberos de España. En Valladolid Web. Recuperado el 16 de julio de 2020, de https://www. valladolidweb.es/valladolid/sucedio/elprimercuerpodebomberos.htm.

Guiard Larrauri, T. (1905). Historia de la noble Villa de Bilbao. Bilbao: Imprenta de José de Astuy.

Gutiérrez Alonso, A. (1997). La hacienda municipal de Burgos en la época Moderna. Los bienes de Propios (1500-1750). Boletín de la Institución Fernán González, 215, pp. 327-354.

Historia de los bomberos. (2020). CurioSfera. Recuperado el 16 de julio de 2020 de https://www.curiosfera.com/historia-bomberos-origen-evolucion.

Iglesias Rouco, L. S. (1978). Arquitectura y urbanismo de Burgos bajo el reformismo ilustrado (1747-1813). Burgos: Caja de Ahorros Municipal de Burgos.

Jarque Martínez, E. (2017). Cuando las cosas van mal. El Concejo y la gestión de sus dificultades (Siglos XVI-XVIII). Zaragoza: Universidad de Zaragoza.

La evolución de los carros de bomberos en EE. UU. (2020). Todos-autos. Recuperado el 15 de julio de 2020, de https://www.todosautos.com/portal/.

Lampérez y Romea, V. (2012). Arquitectura civil española de los siglos I al XVIII. Valladolid: Editorial Maxtor.

López Giménez, E. (2012). Benjamín Franklin y la protección contra incendios, curiosidades de la historia. Recuperado el 15 de julio de 2020, de https:// blog.prefire.es/2012/10/benjamin-franklin-y-la-protección-contra-incendioscuriosidades-de-la-historia.

Muñoz Bort, D. (1986). Fuentes para el estudio de la historia de Huelva: los fondos documentales municipales. Huelva en su historia, 1, pp. 7-28. 
Negueruela Superviola, E. (2011). Historia del alumbrado público en Logroño. Logroño: Instituto de Estudios Riojanos y Ayuntamiento de Logroño.

Riquelme González, R. I. (2011). Benjamin (Ben) Franklin y los Bomberos. El Bombero.cl. Recuperado el 16 de julio de 2020, de https:// elbombero.cl/foro/ threads/benjamin-ben-franklin-y-los-bomberos. 24335.

Rojo 21. Fuego: historia de los incendios (2015). Recuperado el 16 de julio de 2020, de https:// www.rojo21.net/2015/fuego-historia-incendios.html.

Rupérez Almajano, M. N. (1987). La Sociedad Económica de Amigos del País de Soria (1777-1809). Soria: Diputación Provincial de Soria.

Sánchez Domingo, R. (1996). La intendencia de Burgos en el siglo XVIII. Boletín de la Institución Fernán González, 212, pp. 149-168.

Sanz de la Higuera, F. J. (2013). El incendio de las casas de La Calera y el Cabildo Catedral de Burgos (1773-1776). Tiempos Modernos, 27, pp. 1-24.

Sanz de la Higuera, F. J. (2013). La iluminación doméstica en el Burgos del siglo XVIII. ASRI, 5, pp. 1-36.

Sanz de la Higuera, F. J. (2020). El abasto del carbón y de la leña en el Burgos del siglo XVIII. Burgos: Academia Burgense de Historia y Bellas Artes, Institución Fernán González.

Sanz Yagüe, A. I. (2012). La ciudad de Soria en el siglo XVIII. Un estudio sociocultural. Madrid: UNED.

Sanz Yagüe, A. I. (2018). Representación política y participación directa. El "policentrismo» político de Soria y la supervivencia del Común en el siglo XVIII. Madrid: Ediciones Polifemo.

Torres Sánchez, R. (1999). Hacia un irremediable endeudamiento. La hacienda municipal de Cartagena durante el siglo XVIII. En J. L. Pereira Iglesias, J. M. Bernardo Ares y J. M. González Beltrán (coords.), La administración municipal en la Edad Moderna, Actas de la V Reunión Científica de la Asociación Española de Historia Moderna. Cádiz: Asociación Española de Historia Moderna, tomo II, pp. 289-307. https://doi.org/10.20350/ digitalCSIC/11393. 
Vidal Rivas, J. (2012). Incendios, arquitectura y prácticas de poder en el siglo XVIII. Madrid: UNED.

Yuste González, J. (2015). Análisis de los servicios de bomberos. De la épica a la lógica. León: Universidad de León. 